\title{
The IL-8 protease SpyCEP is detrimental for Group A Streptococcus host-cells interaction and biofilm formation
}

\section{Federica Andreoni ${ }^{1}$, Taiji Ogawa ${ }^{1}$, Mariko Ogawa ${ }^{1}$, Jerzy Madon', Satoshi Uchiyama ${ }^{1}$, Reto A. Schuepbach ${ }^{2}$ and Annelies S. Zinkernagel ${ }^{1 *}$}

${ }^{1}$ Division of Infectious Diseases and Hospital Epidemiology, University Hospital Zurich, University of Zurich, Zurich, Switzerland

2 Surgical Intensive Care Medicine, University Hospital Zurich, University of Zurich, Zurich, Switzerland

\section{Edited by:}

Anna Norrby-Teg/und, Karolinska Institutet, Sweden

Reviewed by:

Mattias Collin, Lund University, Sweden

Oliver Goldmann, Helmholtz Centre for Infection Research, Germany

*Correspondence:

Annelies S. Zinkernagel, Division of Infectious Diseases and Hospital

Epidemiology, University Hospital Zurich, University Zurich, Rämistr

100, 8091 Zürich, Switzerland

e-mail: annelies.zinkernagel@usz.ch
SpyCEP-mediated chemokine degradation translates into more efficient spreading and increased severity of invasive Group A Streptococcus (GAS) infections, due to impaired neutrophil recruitment to the site of infection. SpyCEP is markedly up-regulated in invasive as compared to colonizing GAS isolates raising the question whether SpyCEP expression hinders bacterial attachment and thus colonization of the host. To address this question we used a molecular approach involving the use of homologous GAS strains either expressing or not SpyCEP or expressing an enzymatically inactive variant of SpyCEP. We found that expression of enzymatically functional SpyCEP lowered GAS adherence and invasion potential toward various epithelial and endothelial cells. SpyCEP also blunted biofilm formation capacity. Our data indicate that expression of SpyCEP decreases colonization and thus might be detrimental for the spreading of GAS.

Keywords: Group A Streptococcus, SpyCEP, IL-8 protease, colonization, biofilms, virulence factors

\section{INTRODUCTION}

Group A Streptococcus (GAS or Streptococcus pyogenes), a Gram positive bacterium, is a strict human pathogen causing a large burden of disease worldwide. GAS diseases range from mild to severe and life-threatening, resulting in an estimate of 517,000 deaths each year (Carapetis et al., 2005). GAS expresses a vast range of virulence factors that are involved in blunting and evading the host immune system favoring bacterial spreading. Up-regulation of virulence factors is found in invasive GAS isolates and distinguishes them from most colonizing strains (Sumby et al., 2006). When transitioning to invasive strains a distinct phenotype is found in about $40 \%$ of clinical disease-causing isolates. The phenotype is characterized by the up-regulated virulence factors Streptolysin O (SLO), extracellular DNase (Sda1) and Streptococcus pyogenes cell envelope protease (SpyCEP) promoting the spreading of the infection by interfering with recruitment and function of phagocytic cells (Sumby et al., 2006).

One of the most highly up-regulated virulence factors in invasive disease is SpyCEP (Sumby et al., 2006). SpyCEP (aka Spy0416, ScpC, CepA, Prts, or SP24) has the ability to cleave human CXC-chemokines such as GCP-2 (granulocyte chemotactic peptide-2, CXCL6), GRO- $\alpha, \beta, \gamma$ (growth related oncogene$\alpha$, CXCL1, 2, 3), ENA-78 (neutrophil-activating peptide-78, CXCL5), Grap-2 (GRB2-related adapter protein 2) and IL-8 (CXCL8) which correspond to the murine CXC-chemokines MIP-2 and KC (Hidalgo-Grass et al., 2004; Sumby et al., 2006; Chiappini et al., 2012). The presence of a bacterial chemokinedegrading enzyme was first described in a patient suffering from necrotizing fasciitis. Histology revealed a striking paucity of neutrophils despite the massive streptococcal infection and was found to be due to IL-8 degradation by GAS (Hidalgo-Grass et al., 2004).
The protein responsible for IL-8 degradation was later identified as SpyCEP, a subtilisin-like serine protease present in the supernatant of various GAS blood isolates (Edwards et al., 2005). The expression of SpyCEP is regulated by SilCR and CovR/S (HidalgoGrass et al., 2004; Sumby et al., 2008). The protease contains the LPXTG cell wall-anchoring motif (Sumby et al., 2008) present on the cell wall during bacterial log phase growth and shed into the supernatants of bacterial cultures during stationary phase growth (Chiappini et al., 2012). In its mature form SpyCEP is present as a dimer composed of 2 subunits ( 30 and $150 \mathrm{kDa}$ ) generated by intramolecular autocatalytic cleavage. The $30 \mathrm{kDa}$ subunit contains one of the 3 amino acids composing the active site and a stable interaction between the 2 subunits is necessary for enzymatic activity (Zingaretti et al., 2010).

We previously showed that SpyCEP impairs neutrophil endothelial transmigration, promotes resistance to neutrophil killing by reducing the production of Neutrophil Extracellular Traps (NETs) and decreases adherence to and invasion of epithelial Hep-2 cells (Zinkernagel et al., 2008). In in vivo mouse models of invasive and necrotic skin infection, GAS strains expressing SpyCEP caused more severe infections as revealed by the presence of larger skin lesions and increased mortality (Hidalgo-Grass et al., 2006; Zinkernagel et al., 2008). These murine in vivo results are corroborated by clinical findings that invasive clinical GAS isolates display higher SpyCEP activity than the superficial ones (Edwards et al., 2005). However, using GAS WT strains other than the M1T1 strain as well as different mouse and infection models, SpyCEP-deficient mutants were also found to cause larger skin lesions as compared to the SpyCEP-expressing strain (Sjolinder et al., 2008; Sumby et al., 2008). Possibly such divergent findings could be explained by extensive and therefore harmful neutrophil 
recruitment to sites of infection in the absence of SpyCEP and thus causing larger local tissue damage. In an artificial system based on polystyrene beads coated with recombinant SpyCEP, SpyCEP was found to promote uptake of the beads by HUVECs cells (Kaur et al., 2010). No attachment or internalization was however observed for the epithelial cell lines Hep-2 and A549 and SpyCEP did not mediate invasion when heterologously expressed in Group B streptococci (Kaur et al., 2010). This underlines the fact that an artificial system constituted by beads coated with a single protein can act very differently from live bacteria where many more factors present on the cell surface come into play. Up-regulation of most GAS virulence factors has been shown to boost GAS pathogenicity at the time of invasion. However, previous findings describe up-regulation of SpyCEP as detrimental for the colonization of tissue (Zinkernagel et al., 2008). We thus investigated whether the expression of SpyCEP in GAS WT or the lack, thereof using isogenic SpyCEP knockout strains, affected adherence to and invasion of endothelial and epithelial cells. We found that the presence of a functional SpyCEP was detrimental for adherence as well as invasion and that the absence of SpyCEP significantly facilitated biofilm formation.

\section{MATERIALS AND METHODS CELL LINES}

The epithelial cell lines Detroit-562 (human pharyngeal cancer cells) (Peterson et al., 1968) and A549 (adenocarcinomic human alveolar basal epithelial cells) and the primary endothelial cells HUVEC (human umbilical vein endothelial cells, Roche Clonetics) as well as the endothelial cell line Ea.hy926 (immortalized human umbilical vein cells) (Edgell et al., 1983) were used in this work.

\section{GROUP A STREPTOCOCCUS (GAS) STRAINS}

The wild type GAS M1T1 strain 5448 (GAS WT) (Chatellier et al., 2000) was used together with its derivatives GAS $\triangle c e p A$, devoid of SpyCEP expression (Zinkernagel et al., 2008), GAS $\triangle$ cepA compl, in which SpyCEP expression was restored by complementation with the pDestermcepA plasmid (Zinkernagel et al., 2008) and GAS $\Delta$ cepA compl* ${ }^{*}$, expressing an inactive form of SpyCEP (SpyCEP*). GAS $\triangle$ cepA compl* was constructed by transformation of GAS $\triangle$ cepA with the pDestermcep $A^{*}$ plasmid containing a mutated cepA gene (cep $\left.A^{*}\right)$. pDestermcep $A^{*}$ was created by introducing a point mutation exchanging the aspartic acid in position 151 with an alanine in the серA gene contained in pDestermcepA (Zingaretti et al., 2010). The QuickChange XL Site-Directed Mutagenesis kit (Stratagene) was used to introduce the point mutation using the primers: cepA_D151A_fwd GTTGTCGCAGTTATTGCCACA GGGATCGATCCG and cepA_D151A_rev CGGATCGATCCC TGTGGCAATAACTGCGACAAC. The mutation was verified by sequencing and absence of SpyCEP activity was documented using an IL-8 degradation assay, as previously described (Zinkernagel et al., 2008).

\section{GROWTH MEDIA}

A549 and Ea.hy926 cells were grown in Dulbecco's Modified Eagle's Medium (DMEM, Gibco) supplemented with
L-glutamine, Detroit-562 cells were grown in Eagle's minimal essential medium (EMEM, Sigma) and HUVEC cells were grown in Endothelial cell growth medium-2 (EBM-2, Lonza), according to the manufacturer specifications. $10 \%$ fetal calf serum (FBS, PAA) was added to all culture media. GAS strains were grown in Todd-Hewitt broth (THB, BD) medium supplemented with $2 \%$ yeast extract (THY, Oxoid) or on THY-agar plates. Strains bearing the pDCerm-derived plasmids were grown in the presence of $3 \mu \mathrm{g} / \mathrm{ml}$ erythromycin (erm). Phosphate-buffered saline (PBS) was used for washing.

\section{ADHERENCE AND INVASION ASSAYS}

Adherence and invasion assays were carried out as previously described (Uchiyama et al., 2013). Shortly, epithelial and endothelial cells were seeded in 24 well plates. GAS strains were grown $\mathrm{O} / \mathrm{N}$ in $\mathrm{THY}$ medium, diluted 1:10 on the day of the experiment and grown to an OD600 value of 0.4 , at which point they were diluted in cell growth medium $+0.4 \%$ bovine serum albumin (Sigma) and added to the cells at an MOI of 1 or 10 for adherence and invasion, respectively. The cells were then incubated for $30 \mathrm{~min}$ (adherence) or $2 \mathrm{~h}$ (invasion) at $37^{\circ} \mathrm{C}$ and $5 \%$ $\mathrm{CO}_{2}$. For adherence assays the cells were washed $6 \mathrm{X}$ in PBS, trypsinized and lysed with $0.02 \%$ triton-X100. Serial dilutions of the lysates were plated on THY-agar plates and bacterial units enumerated after $\mathrm{O} / \mathrm{N}$ incubation at $37^{\circ} \mathrm{C}$. For invasion assays, the cells were washed $3 \mathrm{X}$ in PBS after $2 \mathrm{~h}$ incubation with GAS followed by incubation in the presence of penicillin and gentamycin (10 and $100 \mu \mathrm{g} / \mathrm{ml}$ ) for $2 \mathrm{~h}$, washed $3 \mathrm{X}$ in PBS and then processed as for the adherence assay.

\section{CONFOCAL LASER SCANNING MICROSCOPY}

The human epithelial cells Detroit-562 were seeded in culture medium into a 24-well microtiter plate containing glass coverslips and incubated for $24-48 \mathrm{~h}$. Bacterial cells were stained using carboxyfluorescein-diacetate-succinimidyl ester (CFSE, Invitrogen), and grown to $\mathrm{OD}_{600}=0.4$. After washing bacteria were added at an MOI of 20. After centrifugation s at $1200 \mathrm{~g}$ for $5 \mathrm{~min}$, the cells were incubated at $37^{\circ} \mathrm{C}$ for $30 \mathrm{~min}$. Nuclei were stained with $4^{\prime}, 6$-diamidino-2-phenylindole (DAPI, Sigma), fixed overnight with $4 \%$ paraformaldehyde and mounted with ProlongGold antifade reagent (Life Technologies, Carlsbad, CA, USA). Confocal microscopy images were acquired using Zeiss LSM 510 META (Carl Zeiss Microscopy GmbH, Jena, Germany) and processed with IMARIS ver. 7.6.1 (Bitplane AG, Zurich, Switzerland). The integrated densities of bacteria on the surface of cell monolayers were evaluated using the analysis-measure tool of the ImageJ software $1.47 \mathrm{v}$.

\section{BIOFILM FORMATION ASSAY}

Biofilm formation was determined using crystal violet staining of adherent biofilm, as previously described (Manetti et al., 2007; Ogawa et al., 2011). Briefly, overnight cultures were grown in THY medium, diluted 10-fold with DMEM and seeded into 96-well plates. Each bacterial strain was seeded into 6 wells and incubated at $37^{\circ} \mathrm{C}$ for $24 \mathrm{~h}$. After removal of medium, the plates were washed 3 times with PBS, and adherent bacteria were stained with $0.2 \%$ crystal violet for $2 \mathrm{~min}$ and washed 3 times with PBS. 
Stained bacteria were eluted with $100 \mu \mathrm{l}$ of $100 \%$ ethanol and the intensity of crystal violet staining was measured by assessing the absorbance at $595 \mathrm{~nm}\left(\mathrm{OD}_{595}\right)$.

\section{SpyCEP* ACTIVITY}

SpyCEP* activity was measured via Western blot as a function of IL-8 degradation, as published (Zinkernagel et al., 2008). An ELISA-based approach was also undertaken to quantify IL-8 degradation. Briefly, $9 \mu \mathrm{l}$ of filtered bacterial supernatants from $\mathrm{OD}_{600} 0.4$ GAS cultures were incubated $\mathrm{O} / \mathrm{N}$ at $37^{\circ} \mathrm{C}$ with $1 \mu \mathrm{l}$ of IL-8 (final concentration $0.1 \mathrm{ng} / \mathrm{ml}$ ) and an ELISA assay (R\&D Systems) was subsequently performed.

\section{SpyCEP-MEDIATED PROTEIN CLEAVAGE ASSAY}

SpyCEP-mediated protein cleavage was carried out using $100 \mathrm{ng} / \mathrm{ml}$ of recombinant SpyCEP in PBS. Recombinant SpyCEP (rSpyCEP) was produced in E. coli as previously described (Kaur et al., 2010).

\section{QUANTIFICATION OF HYALURONIC ACID CONTENT}

Hyaluronic acid was extracted from bacterial pellets in midlogarithmic growth phase as described earlier (Hollands et al., 2010). The samples were diluted and the hyaluronic acid was quantified using a commercial test kit (Corgenix) according to the manufacturer's instructions.

\section{QUANTIFICATION OF ENDOTHELIAL ADHERENCE SURFACE PROTEINS}

In confluent Ea.hy926 cells surface antigens were determined as previously described (Schuepbach et al., 2008). In brief cells were incubated with either buffer alone, rSpyCEP $(10 \mu \mathrm{g} / \mathrm{mL})$ or the same concentration of a control recombinant protein ( $\mathrm{rS} 1 \mathrm{LG}$ ) isolated using the same technique as rSpyCEP, $2 \mathrm{~h}$ before fixation with $2 \% \mathrm{PFA}$ for $30 \mathrm{~min}$ at $4^{\circ} \mathrm{C}$. Followed by addition of murine monoclonal antibodies targeting $\beta$-1-integrin $(\beta$-int), ICAM, VCAM or the endothelial cells integrity markers CD144 and thrombomodulin (TM). A horseradish peroxidase (HRP)coupled goat anti-mouse antibody and tetrametylbenzidine were used for spectrophotometric quantification of specific cell surface antibody binding.

\section{SCANNING ELECTRON MICROSCOPY}

GAS WT and GAS $\triangle$ cepA were cultured to mid-log phase $\left(\mathrm{OD}_{600}=0.4\right)$ and adjusted with $\mathrm{PBS}$ to $2 \times 10^{9} \mathrm{CFU} / \mathrm{ml}$, followed by fixation with $2.5 \%$ glutaraldehyde for $24 \mathrm{~h}$ at $4^{\circ} \mathrm{C}$ followed by placement on cover glass slide by cytospin at $1000 \mathrm{~g}$ for 3 min. Bacterial samples were postfixed with $1 \%$ osmium tetroxide followed by dehydration with increasing concentrations of ethanol (70-100\%). After critical point drying (Bal Tec CPD 030 Critical point dryer, Leica Biosystems), the samples were coated with platinum using sputter-coating system (Bal Tec SCD 500 Sputter Coater, Leica Biosystems) and then examined with a field emission scanning electron microscopy (Supra 50 VP, Carl Zeiss) using inlens detector.

\section{STATISTICS}

All statistical analyses were performed using a two-tailed unpaired $t$-test.

\section{RESULTS \\ ENHANCED GAS ADHERENCE TO AND INVASION OF EPITHELIAL AND ENDOTHELIAL CELLS IN THE ABSENCE OF SPYCEP}

SpyCEP is barely expressed by superficial but largely induced in invasive GAS strains (Sumby et al., 2006). We thus wondered whether the virulence factor SpyCEP might influence the adherence and invasion potential of GAS in epithelial and endothelial cells. In order to test this we used the highly invasive M1T1 strain expressing SpyCEP (Zinkernagel et al., 2008) and found that in its isogenic SpyCEP-deficient counterpart, GAS $\triangle$ cepA, adherence to and invasion of epithelial as well as endothelial cells was significantly enhanced (Figures 1A,B). Complementation of GAS $\triangle$ cepA with a plasmid containing the cepA gene (GAS $\triangle c e p A$ compl) restored the wild type phenotype resulting in lower adherence and invasion.

\section{REDUCED ADHERENCE AND INVASION POTENTIAL DUE TO LOSS IN SpyCEP ACTIVITY}

In order to investigate whether SpyCEP activity affected adherence and invasion, GAS $\triangle c e p A$ was transformed with a plasmid containing a mutated version of SpyCEP devoid of serine protease activity (GAS $\triangle c e p A$ compl $^{*}$ ). SpyCEP* expression was verified by binding studies using anti-SpyCEP antiserum (Figure S1A). Loss of SpyCEP activity in GAS $\triangle c e p A$ compl* $^{*}$ was assessed as a measure of IL-8 degradation both via ELISA and Western blot (Figure S1B). The adherence and invasion potential of GAS $\triangle c e p A$ compl* $^{*}$ was tested on Detroit-562 cells as representative epithelial cells and on Ea.hy926 cells as representative endothelial cells. Similarly to the GAS $\Delta c e p A$ strain adherence and invasion of GAS $\triangle c e p A$ compl $^{*}$ was found to be significantly higher compared to GAS WT suggesting that SpyCEP activity per se impedes GAS adherence and invasion (Figures 2A,B).

\section{ENHANCED ATTACHMENT AND BIOFILM FORMATION IN THE ABSENCE OF SpyCEP}

The attachment of the GAS strains to Detroit-562 cells was quantified by confocal fluorescence microscopy showing higher attachment in the absence of SpyCEP protein or in the presence of an inactive SpyCEP version (Figures 3A,B). Enhanced cellular invasion, efficient colonization and persistence of bacteria have been linked to biofilm-forming capacity (Sekhar et al., 2009; Ogawa et al., 2011). We thus investigated in a next step whether the absence of SpyCEP would influence biofilm formation and thereby alter GAS colonization potential. We found biofilm formation to increase when SpyCEP was not present or non-functional (Figure 3C).

\section{SpyCEP ACTIVITY DOES NOT INFLUENCE ADHESION MOLECULES STRUCTURE AND EXPRESSION}

To exclude that SpyCEP hindered bacterial adherence by cleavage of fibrinogen, fibronectin, or the M1 protein, among the main factors involved in GAS adherence to host cells, we performed cleavage assays. We found that none of these host or bacterial proteins was cleaved by rSpyCEP (Figure 4A). To reveal other possible mechanisms by which SpyCEP activity could lead to reduced adherence and invasion of host cells, a cell-surface 


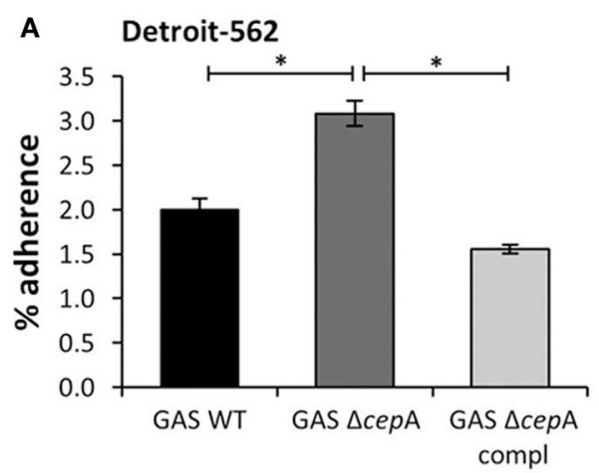

A549

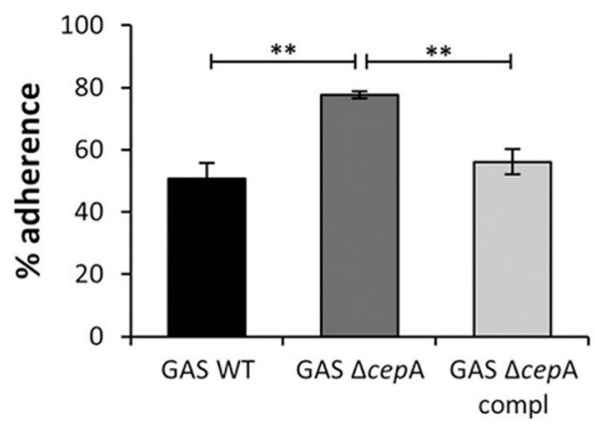

Ea.yh926

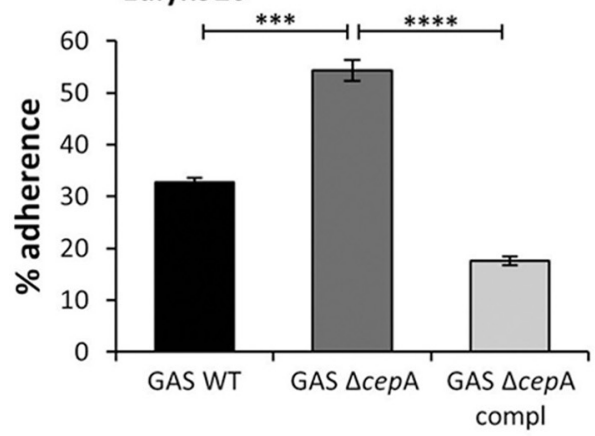

HUVEC

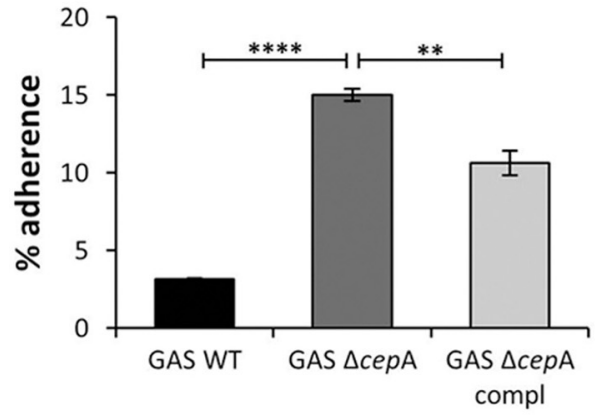

FIGURE 1 | Enhanced GAS adherence to and invasion of epithelial and endothelial cells in the absence of SpyCEP. The epithelial cell lines

Detroit-562 and A549 and the endothelial cells Ea.hy926 and HUVECs were challenged with the GAS strains GAS WT, GAS $\triangle$ cepA, and GAS $\triangle$ cepA compl. MOI of 1 was used for adherence assays and $\mathrm{MOI}$ of 10 was used for invasion assays. To assess adherence and invasion an antibiotic protection assay was used and adhering/invading bacteria enumerated by plating and

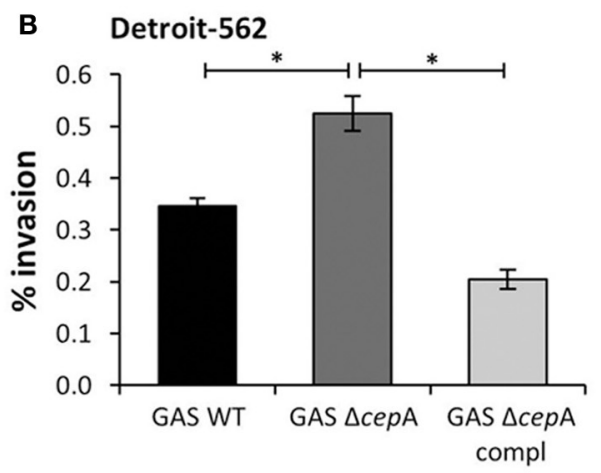

A549

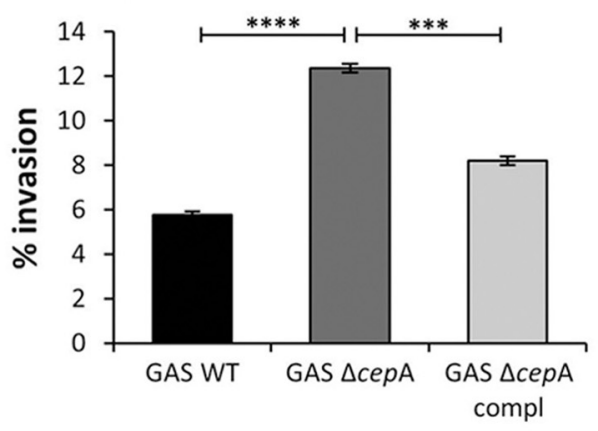

EA.yh926

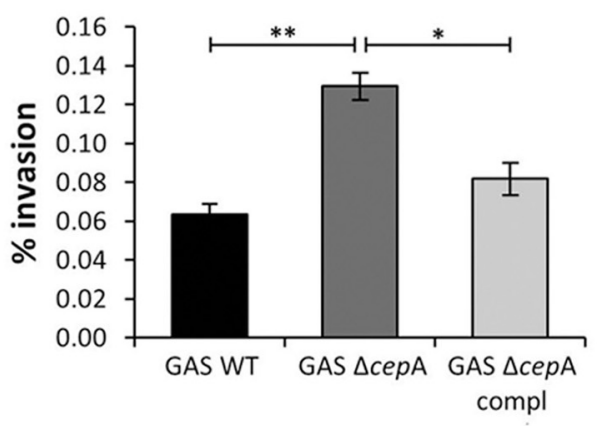

HUVEC

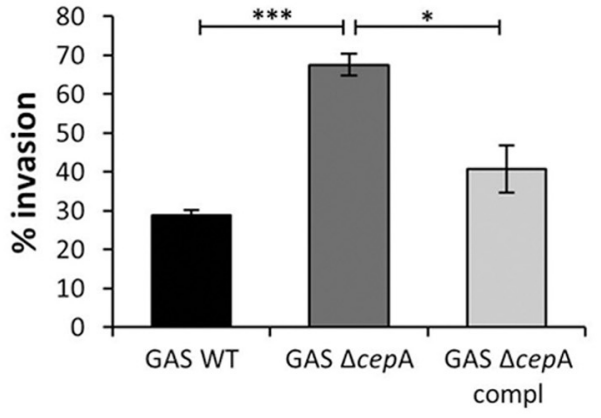

colony counting. (A) GAS adherence to and (B) invasion of the epithelial and endothelial cells were assessed after $30 \mathrm{~min}$ and $2 \mathrm{~h}$ respectively. The absence of SpyCEP significantly increased adherence to and invasion of epithelial and endothelial cells by GAS. Each experiment was carried out in triplicate; the figure shows one representative experiment. Error bars represent the standard error, statistical analysis was carried out using a two-tailed $t$-test (ns $=p>0.05,{ }^{*} p \leq 0.05,{ }^{* *} p \leq 0.01,{ }^{* * *} p \leq 0.001,{ }^{* * * *} p<0.0001$ ). 

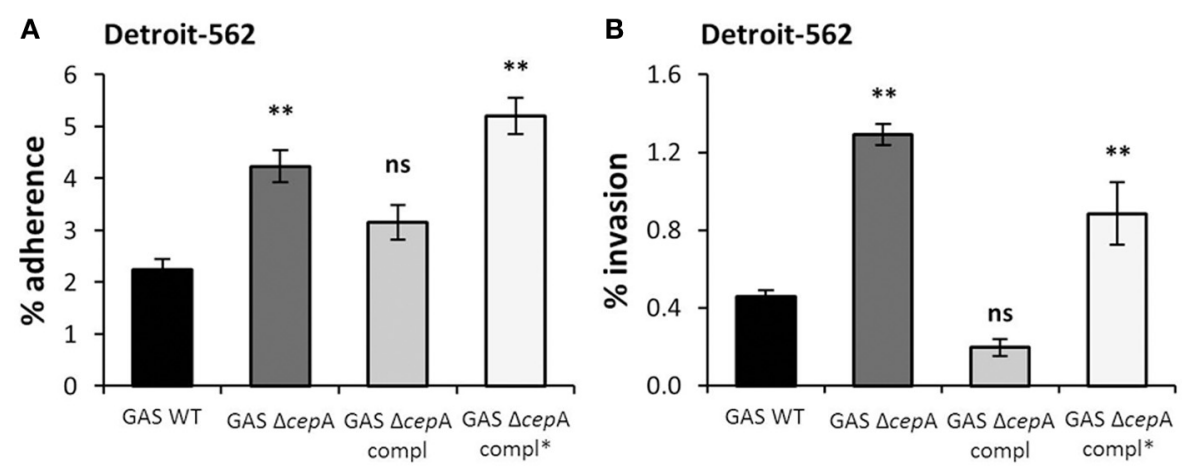

EA.yh926

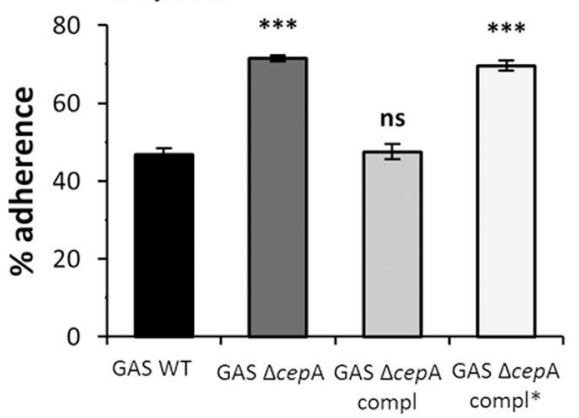

EA.yh926

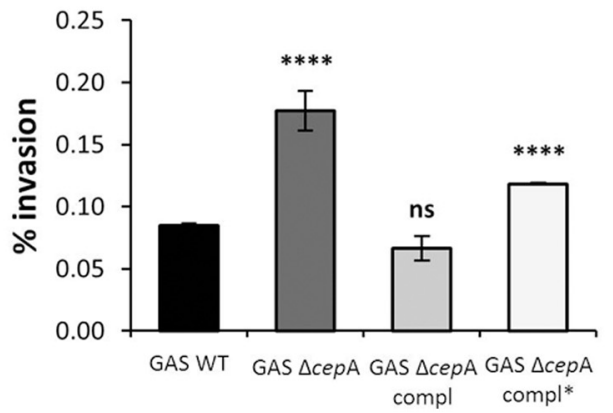

FIGURE 2 | SpyCEP activity requirement for GAS adherence to and invasion of epithelial and endothelial cells. Representative epithelial (Detroit-562) and endothelial (EA.hy926) cell lines were challenged with GAS WT, GAS $\triangle$ cepA, GAS $\triangle$ cepA compl and GAS $\triangle$ cepA compl*.

(A) Adherence and (B) invasion assays were carried out as described in Figure 1. GAS $\triangle$ cepA compl* behaved like GAS $\triangle$ cepA in terms of

adherence and invasion to Detroit-562 and EA.hy926 cells. Each experiment was carried out in triplicate and the graphs show one representative experiment. Error bars represent the standard error, statistical analysis was carried out using a two-tailed $t$-test (ns $=p>0.05,{ }^{*} p \leq 0.05,{ }^{* *} p \leq 0.01,{ }^{* * *} p \leq 0.001,{ }^{* * * *} p<0.0001$ ). The $p$-values are relative to GAS WT.

ELISA targeting the host adhesion molecule $\beta$ - 1 integrin was performed on the endothelial cell line Ea.hy926 in the presence or absence of rSpyCEP or an unrelated protein (rS1LG) purified using the same method. $\beta-1$ integrin can interact with fibronectin and therefore mediate attachment of GAS (Ruoslahti, 1991). No difference in the $\beta$ - 1 integrin amount present on the host cells surface upon exposure to rSpyCEP was observed (Figure 4B). The same result was obtained for the adhesion molecules VCAM and ICAM and for the endothelial cells integrity markers VE-cadherin (CD144) and thrombomodulin (TM) (Figure S2A). Scanning electron microscopy showed similar surface quality (Figure S3). Hyaluronic acid capsule content influences adherence (Cho and Caparon, 2005; Hollands et al., 2010). Thus we quantified the hyaluronic acid content of the various GAS strains and again found no difference (Figure 4C). It can therefore be concluded that SpyCEP-mediated modification of the aforementioned factors is not responsible for variations in the colonization potential of GAS $\triangle c e p A$.

\section{DISCUSSION}

We show that expression of functional SpyCEP is detrimental for adherence to and invasion of human epithelial and endothelial cells as well as for biofilm formation, underscoring the importance of virulence factors regulation for bacterial pathogenesis. Our findings in GAS M1T1 support that SpyCEP interferes with host cell adherence and invasion and thus impedes GAS colonization. Our data is consistent with epidemiologic studies reporting that superficial GAS strains in general barely express SpyCEP (Sumby et al., 2006). In colonization and superficial infections bacterial virulence factors are usually not highly expressed which, according to the trade-off hypothesis, allows the bacterium to multiply and persist within the host without causing too much damage allowing perpetuation of the species (Wollein Waldetoft and Raberg, 2014). Evolutionary speaking, the expression of virulence factors triggered in severe invasive diseases should therefore be an obstacle to the maintenance and spreading of the colonizing GAS population. Thus GAS has evolved a strategy to maintain the expression of virulence factors while colonizing and to allow their expression only under particular conditions, e.g., invasive outbursts. The two component regulatory system $\operatorname{cov} R S$ allows down-regulation of GAS virulence factors ensuring a balance between bacterial spreading and host survival. During invasive infections however, the expression of virulence factors is essential for the survival of GAS; from here their maintenance in the genome and the possibility to up-regulate their expression level according to the situation.

Previous reports found that upon invasion SpyCEP is among the most strongly up-regulated virulence factors in GAS due to a point mutation in $\operatorname{cov} R S$ resulting in de-repression of virulence genes (Sumby et al., 2006). Consistently, SpyCEP has been 


\section{A Confocal fluorescence microscopy}

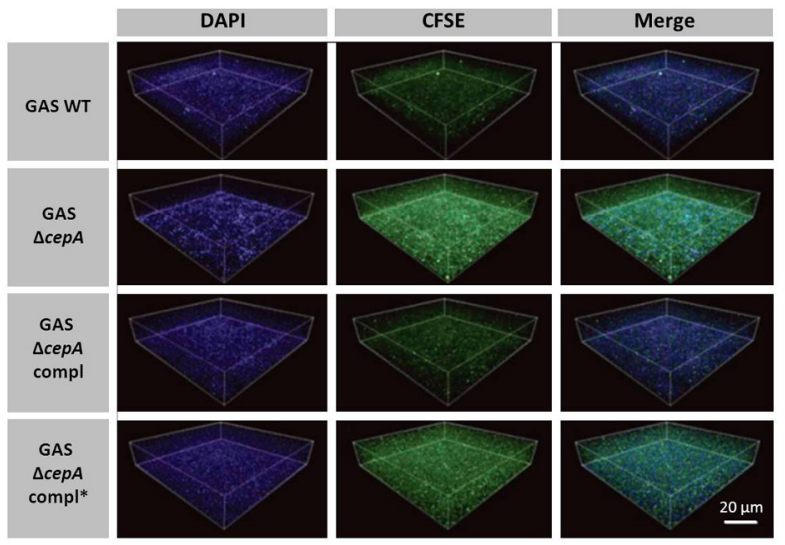

B Integrated density

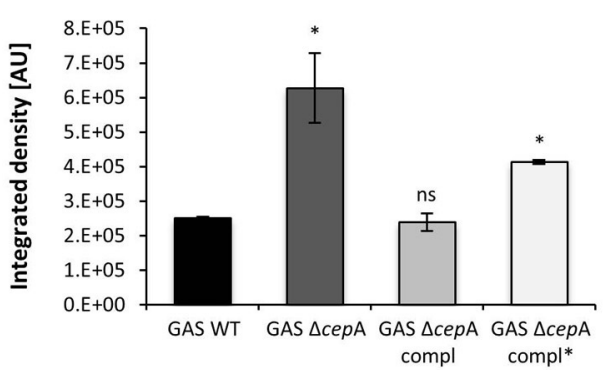

FIGURE 3 | Decreased GAS cell surface attachment and biofilm formation in the presence of SpyCEP. (A) Microscopy analysis of GAS attached to the surface of human pharyngeal cells. GAS strains and Detroit-562 cells were stained with CFSE (green) and DAPI (blue) respectively and attachment was assessed using a fluorescence confocal laser scanning microscope. The scale bar in the confocal images represents $20 \mu \mathrm{m}$.

(B) Integrated densities of attached bacteria obtained from confocal images were evaluated using ImageJ. The integrated density is measured in arbitrary

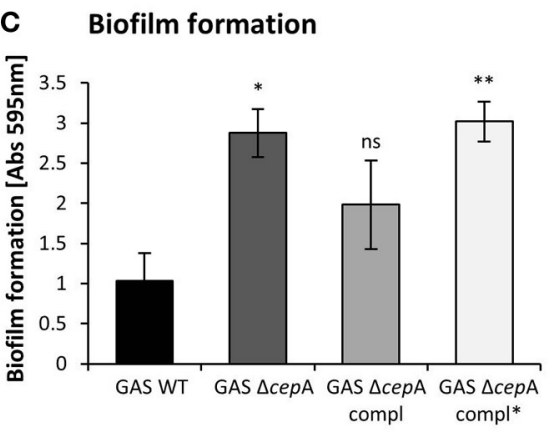

units (AU). Data were pooled from three independent experiments done in triplicate, mean $\pm S D ;{ }^{*} p<0.01$, compared to GAS WT. (C) Biofilm formation was assessed for GAS WT, GAS $\triangle$ cepA, GAS $\Delta$ cepA compl and GAS $\Delta$ cepA compl*. The absence of SpyCEP or the presence of an inactive SpyCEP form resulted in increased biofilm formation. The graph represents pooled data from three independent experiments; the error bars represent the standard deviation. Statistical analysis was carried out using a two-tailed $t$-test (ns $=p>0.05, * p \leq 0.05, * * \leq 0.01$ ). The $p$-values are relative to GAS WT. highlighted for boosting virulence in invasive disease through IL-8 degradation thereby inhibiting neutrophil migration and bacterial killing (Zinkernagel et al., 2008). Inactivation of the $\operatorname{cov} R S$ virulence regulator results in higher expression of various bacterial virulence factors, including SpyCEP, without affecting fitness cost in invasive infections but hindering colonization (Alam et al., 2013). In accordance, in a murine naso-pahryngeal infection model, inactivation of the $\operatorname{cov} R S$ virulence regulator was reported to impair shedding and transmission of bacteria by the mice (Alam et al., 2013). Also, a shorter naso-pharingeal carriage was shown for bacteria possessing a functional rocA gene able to increase $\operatorname{cov} R$ transcription and therefore able to enhance covRS-regulated virulence factors transcription, including SpyCEP (Lynskey et al., 2013). All these findings suggest that the presence of SpyCEP might lead to a loss in colonization potential.

We show that SpyCEP indeed interferes with GAS colonization of epithelial cell lines, representing the lining of the major sites of GAS colonization in humans such as the oropharynx and the lungs (Aronoff and Mulla, 2008). We used a molecular approach to test whether SpyCEP expression was required for interference with colonization of eukaryotic cells. Consistent with our conclusion the isogenic GAS $\triangle c e p A$ strain, deficient in SpyCEP expression and thus comparable to a colonizing strain, possessed a much higher adherence and invasion potential than its GAS WT counterpart. Complementation of GAS $\Delta c e p A$ with a plasmid containing the SpyCEP gene restored the phenotype of the invasive GAS WT strain further supporting our conclusion that SpyCEP affects the ability of GAS to colonize both epithelial and endothelial cells.

Biofilm formation promotes bacterial survival and persistence on living and non-living surfaces. We found that GAS WT attached less to Detroit-562 cell layers and formed less biofilms compared to its isogenic SpyCEP mutant. Complementation of GAS $\triangle c e p A$ to restore SpyCEP expression again restored the wild type phenotype. To our knowledge this is the first report highlighting the involvement of SpyCEP in modulating biofilm formation. 


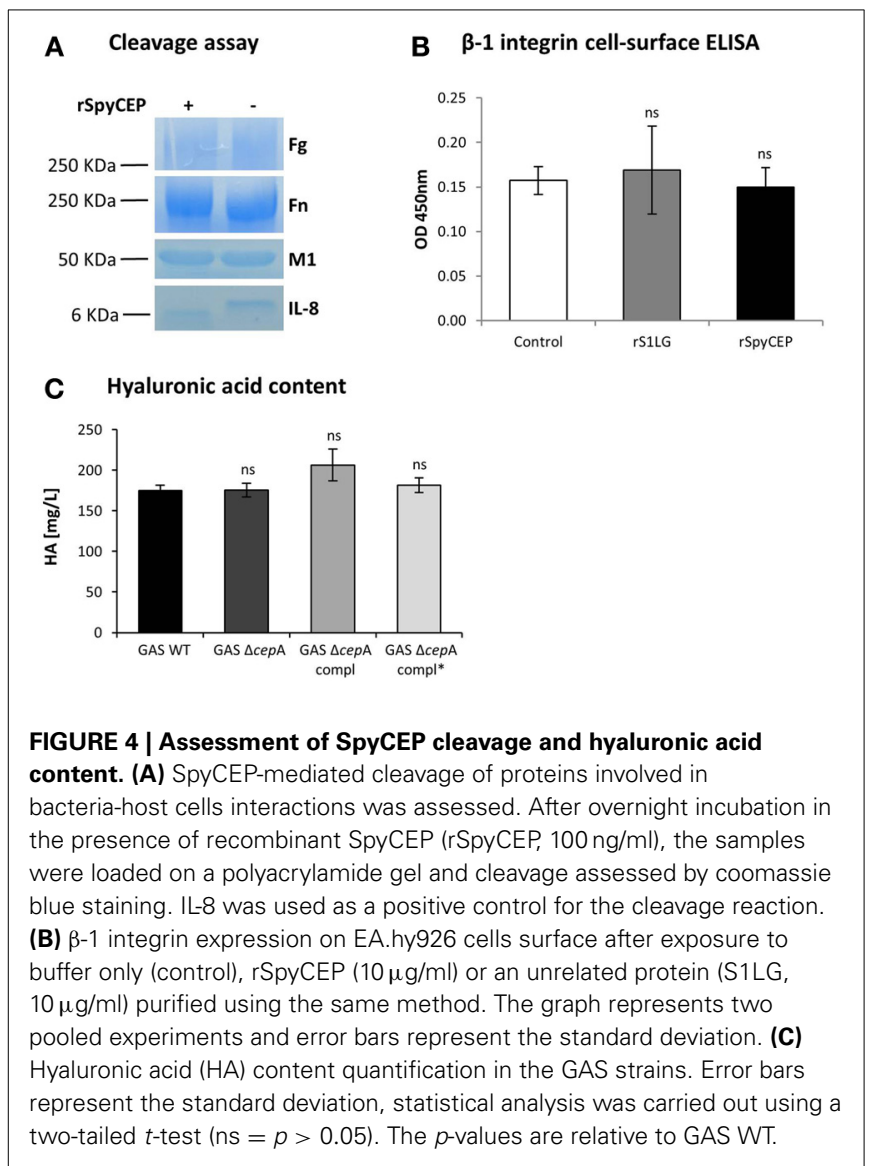

To better understand whether SpyCEP enzymatic activity per se influences host cells colonization we examined its role in colonization. SpyCEP is a serine protease and thus it seemed likely that the protease activity would be causative. To test whether enzymatic or structural properties of SpyCEP interfere with GAS colonization we engineered the active site mutant SpyCEP* as published by Zingaretti et al. (2010). We found that heterologous expression of SpyCEP* resulted in the same phenotype as lack of SpyCEP expression whereas heterologous expression of wild type SpyCEP restored the wild type phenotype pointing toward SpyCEP activity as a possible cause for impaired colonization. As previously described, the active form of SpyCEP undergoes maturation through autocatalytic selfcleavage that gives rise to two fragments of 30 and $150 \mathrm{kDa}$ containing one and two of the amino acids composing the catalytic triad of the enzyme's active site respectively (Zingaretti et al., 2010). SpyCEP activity depends on the correct assembly of these two fragments which leads to the formation of an active enzyme. Although the secondary structure of SpyCEP* was reported to be very similar to the one of wild type SpyCEP (Zingaretti et al., 2010), correct assembly of the two subunits will not occur in GAS $\triangle$ cepA compl* as a result of lack of SpyCEP auto-cleavage. Based on these considerations we hypothesized that loss of enzyme function results in missassembly of SpyCEP* resulting in turn in a structure that either influences bacteria-host cell interactions or disables cleavage of factors important for GAS adherence, invasion and biofilm formation.

To determine whether the absence of SpyCEP activity or the absence of a correctly folded enzyme would be causative for the observed increase in colonization potential, we set up a series of cleavage assays involving SpyCEP and some of the main factors involved in GAS adherence to host cells. Potential target substrates of SpyCEP activity are bacterial virulence factors and host cell receptors (Ender et al., 2013) or bacterial binding targets. Among the main bacterial factors known to modulate GAS ability to colonize the host, we found that SpyCEP did not cleave the M1 protein or affect hyaluronic acid content. M1 was shown to be the major factor present on the GAS surface mediating binding to host cells through interaction with fibrinogen (Uchiyama et al., 2013). A very high content in hyaluronic acid capsule is deemed responsible for decreased bacterial attachment to host cells possibly due to masking of bacterial surface adhesins (Hollands et al., 2010) although the effect seems to be serotype-dependent as encapsulation was shown not to be detrimental for pharyngeal infection for a emm18 GAS strain (Lynskey et al., 2013). On the host's side we found that neither fibrinogen nor fibronectin, two of the host proteins known to mediate adherence, were cleaved by SpyCEP. The amount of $\beta-1$ integrin found on the host cell surface was also not affected by SpyCEP activity clearly indicating that the disruption of the fibonectin-mediated interaction between GAS and host cells is not the cause of loss in cell attachment. Indirect effects of SpyCEP via cleavage of IL-8 seemed unlikely, since the addition of exogenous IL-8 did not influence adherence and invasion in our hands (data not shown). Previously we also showed that the family of mammalian protease sensing receptors, the proteaseactivated receptors PAR, was not activated by SpyCEP, in contrast to other GAS proteases, excluding also this pathway from the possible mechanisms mediating modulation of GAS colonization abilities (Ender et al., 2013).

GAS interaction with host cells is mediated by a vast variety of molecules, called adhesins, present on the cell surface that interact with various types of receptors present on the host cells (for an extensive review see Nobbs et al., 2009). Although we screened a few of these factors to find possible ways by which SpyCEP could interfere with host cells adherence and invasion, a comprehensive screening would prove very challenging. The first step involved in the adherence of GAS to host cells is mediated by electrostatic interactions between bacterial and host cell surfaces followed by the establishment of stronger bonds between adhesins and host cell receptors (Hasty et al., 1992). Changes in the electrostatic charge on the bacterial surface could therefore interfere with GAS adherence to host cells. The presence of the active form of SpyCEP on the GAS surface could be acting by altering electrostatic charges therefore undermining the establishment of the first contact with the host cells. Another way by which the protease activity of SpyCEP might interfere with adherence is by hindering the action of bacterial sortases, i.e., proteins that mediate the exposure of adhesins to the bacterial cell surface allowing for interactions with host cells to happen (Marraffini et al., 2006). Our data on adherence, invasion and biofilm formation strongly suggest that the expression of functional SpyCEP is detrimental for promoting colonization of 
tissues and, in the long run, detrimental for the persistence and subsequent spreading of a superficial infection. Taken together our work illustrates the importance of bacterial virulence factor regulation and how this influences disease. The GAS virulence factor SpyCEP is down-regulated during colonization since it is detrimental for colonization but up-regulated upon invasion where it boosts virulence in invasive disease.

\section{ACKNOWLEDGMENTS}

This work was supported by grants from the Swiss National Science Foundation (31-130748 to Annelies S. Zinkernagel and PZ00P3_136639 to Reto A. Schuepbach), funds from the "Zentrum für Klinische Forschung" and by the "Stiftung für Medizinische Forschung, University of Zurich." We thank Shiranee Sriskandan (Imperial College, London, UK) for kindly providing the polyclonal anti-SpyCEP antibody, Wolfgang Thormann (Clinical Pharmacology Laboratory, Institute for Infectious Diseases, Bern, Switzerland) for performing the quantification of hyaluronic acid and Andrea Tarnutzer for critical reading of the manuscript. The authors acknowledge the assistance and support of the Center for Microscopy and Image Analysis, University of Zurich for performing confocal and scanning electron microscopy experiments.

\section{SUPPLEMENTARY MATERIAL}

The Supplementary Material for this article can be found online at: http://www.frontiersin.org/journal/10.3389/fmicb. 2014.00339/abstract

Figure S1 | Expression and activity of SpyCEP*. (A) FACS analysis of anti-SpyCEP antiserum binding to various GAS strains' surface. (B) Quantification of SpyCEP activity in the supernatants of the various GAS strains by assessment of IL-8 degradation both via ELISA (top panel) and Western blot (bottom panel). Error bars represent standard deviation, statistical analysis was carried out using a two-tailed $t$-test (ns $=p>0.05$ $\left.{ }^{* *} p \leq 0.01,{ }^{* * *} p \leq 0.001,{ }^{* * * *} p<0.0001\right)$.

Figure S2 | Assessment of cell-surface host proteins by ELISA. (A) Surface expression of the adhesion molecules ICAM and VCAM and of the endothelial cells integrity markers CD144 and trombomodulin (TM) on EA.hy926 cells after exposure to buffer only (control), rSpyCEP $(10 \mu \mathrm{g} / \mathrm{ml})$ or an unrelated protein $(\mathrm{S} 1 \mathrm{LG}, 10 \mu \mathrm{g} / \mathrm{ml})$ purified using the same method. The graph represents two pooled experiments and error bars represent the standard deviation.

Figure S3 | Scanning electron microscopy of GAS WT (A) and GAS $\Delta$ cepA (B). Mid-log phase bacterial culture was fixed on cover glass. Each strain observed with FE-SEM displayed the surface structure and both of them had similar surface quality. Scale bar represents $100 \mathrm{~nm}$.

\section{REFERENCES}

Alam, F. M., Turner, C. E., Smith, K., Wiles, S., and Sriskandan, S. (2013). Inactivation of the CovR/S virulence regulator impairs infection in an improved murine model of Streptococcus pyogenes naso-pharyngeal infection. PLoS ONE 8:e61655. doi: 10.1371/annotation/1144e132-9e69-47bb-8e65-1414dbb01db7

Aronoff, D. M., and Mulla, Z. D. (2008). Postpartum invasive group A streptococcal disease in the modern era. Infect. Dis. Obstet. Gynecol. 2008:796892. doi: $10.1155 / 2008 / 796892$

Carapetis, J. R., Steer, A. C., Mulholland, E. K., and Weber, M. (2005). The global burden of group A streptococcal diseases. Lancet Infect. Dis. 5, 685-694. doi: 10.1016/S1473-3099(05)70267-X
Chatellier, S., Ihendyane, N., Kansal, R. G., Khambaty, F., Basma, H., NorrbyTeglund, A., et al. (2000). Genetic relatedness and superantigen expression in group A streptococcus serotype M1 isolates from patients with severe and nonsevere invasive diseases. Infect. Immun. 68, 3523-3534. doi: 10.1128/IAI.68.6.3523-3534.2000

Chiappini, N., Seubert, A., Telford, J. L., Grandi, G., Serruto, D., Margarit, I., et al. (2012). Streptococcus pyogenes SpyCEP influences host-pathogen interactions during infection in a murine air pouch model. PLoS ONE 7:e40411. doi: 10.1371/journal.pone.0040411

Cho, K. H., and Caparon, M. G. (2005). Patterns of virulence gene expression differ between biofilm and tissue communities of Streptococcus pyogenes. Mol. Microbiol. 57, 1545-1556. doi: 10.1111/j.1365-2958.2005.04786.x

Edgell, C. J., Mcdonald, C. C., and Graham, J. B. (1983). Permanent cell line expressing human factor VIII-related antigen established by hybridization. Proc. Natl. Acad. Sci. U.S.A. 80, 3734-3737. doi: 10.1073/pnas.80. 12.3734

Edwards, R. J., Taylor, G. W., Ferguson, M., Murray, S., Rendell, N., Wrigley, A., et al. (2005). Specific C-terminal cleavage and inactivation of interleukin- 8 by invasive disease isolates of Streptococcus pyogenes. J. Infect. Dis. 192, 783-790. doi: $10.1086 / 432485$

Ender, M., Andreoni, F., Zinkernagel, A. S., and Schuepbach, R. A. (2013). Streptococcal SpeB Cleaved PAR-1 Suppresses ERK Phosphorylation and Blunts Thrombin-Induced Platelet Aggregation. PLoS ONE 8:e81298. doi: 10.1371/journal.pone.0081298

Hasty, D. L., Ofek, I., Courtney, H. S., and Doyle, R. J. (1992). Multiple adhesins of streptococci. Infect. Immun. 60, 2147-2152.

Hidalgo-Grass, C., Dan-Goor, M., Maly, A., Eran, Y., Kwinn, L. A., Nizet, V., et al. (2004). Effect of a bacterial pheromone peptide on host chemokine degradation in group A streptococcal necrotising soft-tissue infections. Lancet 363, 696-703. doi: $10.1016 / \mathrm{S} 0140-6736(04) 15643-2$

Hidalgo-Grass, C., Mishalian, I., Dan-Goor, M., Belotserkovsky, I., Eran, Y., Nizet, V., et al. (2006). A streptococcal protease that degrades CXC chemokines and impairs bacterial clearance from infected tissues. EMBO J. 25, 4628-4637. doi: 10.1038/sj.emboj.7601327

Hollands, A., Pence, M. A., Timmer, A. M., Osvath, S. R., Turnbull, L., Whitchurch, C. B., et al. (2010). Genetic switch to hypervirulence reduces colonization phenotypes of the globally disseminated group A streptococcus M1T1 clone. J. Infect. Dis. 202, 11-19. doi: 10.1086/653124

Kaur, S. J., Nerlich, A., Bergmann, S., Rohde, M., Fulde, M., Zahner, D., et al. (2010). The CXC chemokine-degrading protease SpyCep of Streptococcus pyogenes promotes its uptake into endothelial cells. J. Biol. Chem. 285, 27798-27805. doi: 10.1074/jbc.M109.098053

Lynskey, N. N., Goulding, D., Gierula, M., Turner, C. E., Dougan, G., Edwards, R. J., et al. (2013). RocA truncation underpins hyper-encapsulation, carriage longevity and transmissibility of serotype M18 group A streptococci. PLoS Pathog. 9:e1003842. doi: 10.1371/journal.ppat.1003842

Manetti, A. G., Zingaretti, C., Falugi, F., Capo, S., Bombaci, M., Bagnoli, F., et al. (2007). Streptococcus pyogenes pili promote pharyngeal cell adhesion and biofilm formation. Mol. Microbiol. 64, 968-983. doi: 10.1111/j.13652958.2007.05704.x

Marraffini, L. A., Dedent, A. C., and Schneewind, O. (2006). Sortases and the art of anchoring proteins to the envelopes of gram-positive bacteria. Microbiol. Mol. Biol. Rev. 70, 192-221. doi: 10.1128/MMBR.70.1.192-221.2006

Nobbs, A. H., Lamont, R. J., and Jenkinson, H. F. (2009). Streptococcus adherence and colonization. Microbiol. Mol. Biol. Rev. 73, 407-450. doi: 10.1128/MMBR.00014-09

Ogawa, T., Terao, Y., Okuni, H., Ninomiya, K., Sakata, H., Ikebe, K., et al. (2011). Biofilm formation or internalization into epithelial cells enable Streptococcus pyogenes to evade antibiotic eradication in patients with pharyngitis. Microb. Pathog. 51, 58-68. doi: 10.1016/j.micpath.2011.03.009

Peterson, W. D. Jr., Stulberg, C. S., Swanborg, N. K., and Robinson, A. R. (1968). Glucose-6-phosphate dehydrogenase isoenzymes in human cell cultures determined by sucrose-agar gel and cellulose acetate zymograms. Proc. Soc. Exp. Biol. Med. 128, 772-776. doi: 10.3181/00379727-128-33119

Ruoslahti, E. (1991). Integrins. J. Clin. Invest. 87, 1-5. doi: 10.1172/JCI114957

Schuepbach, R. A., Feistritzer, C., Brass, L. F., and Riewald, M. (2008). Activated protein C-cleaved protease activated receptor-1 is retained on the endothelial cell surface even in the presence of thrombin. Blood 111, 2667-2673. doi: 10.1182/blood-2007-09-113076 
Sekhar, S., Kumar, R., and Chakraborti, A. (2009). Role of biofilm formation in the persistent colonization of Haemophilus influenzae in children from northern India. J. Med. Microbiol. 58, 1428-1432. doi: 10.1099/jmm.0. 010355-0

Sjolinder, H., Lovkvist, L., Plant, L., Eriksson, J., Aro, H., Jones, A., et al. (2008). The ScpC protease of Streptococcus pyogenes affects the outcome of sepsis in a murine model. Infect. Immun. 76, 3959-3966. doi: 10.1128/IAI.00128-08

Sumby, P., Whitney, A. R., Graviss, E. A., Deleo, F. R., and Musser, J. M. (2006). Genome-wide analysis of group a streptococci reveals a mutation that modulates global phenotype and disease specificity. PLoS Pathog. 2:e5. doi: 10.1371/journal.ppat.0020005

Sumby, P., Zhang, S., Whitney, A. R., Falugi, F., Grandi, G., Graviss, E. A., et al. (2008). A chemokine-degrading extracellular protease made by group A Streptococcus alters pathogenesis by enhancing evasion of the innate immune response. Infect. Immun. 76, 978-985. doi: 10.1128/IAI.01354-07

Uchiyama, S., Andreoni, F., Zurcher, C., Schilcher, K., Ender, M., Madon, J., et al. (2013). Coiled-coil irregularities of the M1 protein structure promote M1-fibrinogen interaction and influence group A Streptococcus host cell interactions and virulence. J. Mol. Med. (Berl.) 91, 861-869. doi: 10.1007/s00109013-1012-6

Wollein Waldetoft, K., and Raberg, L. (2014). To harm or not to harm? On the evolution and expression of virulence in group A streptococci. Trends Microbiol. 22, 7-13. doi: 10.1016/j.tim.2013.10.006

Zingaretti, C., Falugi, F., Nardi-Dei, V., Pietrocola, G., Mariani, M., Liberatori, S., et al. (2010). Streptococcus pyogenes SpyCEP: a chemokine-inactivating protease with unique structural and biochemical features. FASEB J. 24, 2839-2848. doi: 10.1096/fj.09-145631

Zinkernagel, A. S., Timmer, A. M., Pence, M. A., Locke, J. B., Buchanan, J. T., Turner, C. E., et al. (2008). The IL-8 protease SpyCEP/ScpC of group A Streptococcus promotes resistance to neutrophil killing. Cell Host Microbe 4, 170-178. doi: 10.1016/j.chom.2008.07.002

Conflict of Interest Statement: The authors declare that the research was conducted in the absence of any commercial or financial relationships that could be construed as a potential conflict of interest.

Received: 23 April 2014; accepted: 18 June 2014; published online: 10 July 2014. Citation: Andreoni F, Ogawa T, Ogawa M, Madon J, Uchiyama S, Schuepbach RA and Zinkernagel AS (2014) The IL-8 protease SpyCEP is detrimental for Group A Streptococcus host-cells interaction and biofilm formation. Front. Microbiol. 5:339. doi: $10.3389 /$ fmicb.2014.00339

This article was submitted to Infectious Diseases, a section of the journal Frontiers in Microbiology.

Copyright (c) 2014 Andreoni, Ogawa, Ogawa, Madon, Uchiyama, Schuepbach and Zinkernagel. This is an open-access article distributed under the terms of the Creative Commons Attribution License (CC BY). The use, distribution or reproduction in other forums is permitted, provided the original author(s) or licensor are credited and that the original publication in this journal is cited, in accordance with accepted academic practice. No use, distribution or reproduction is permitted which does not comply with these terms. 